\title{
$\alpha$-Coupled Fixed Points and Their Application in Dynamic Programming
}

\author{
J. Harjani, J. Rocha, and K. Sadarangani \\ Departamento de Matemáticas, Universidad de Las Palmas de Gran Canaria, Campus de Tafira Baja, \\ 35017 Las Palmas de Gran Canaria, Spain
}

Correspondence should be addressed to J. Harjani; jharjani@dma.ulpgc.es

Received 17 June 2014; Accepted 26 July 2014; Published 19 August 2014

Academic Editor: Erdal Karapinar

Copyright (C) 2014 J. Harjani et al. This is an open access article distributed under the Creative Commons Attribution License, which permits unrestricted use, distribution, and reproduction in any medium, provided the original work is properly cited.

We introduce the definition of $\alpha$-coupled fixed point in the space of the bounded functions on a set $S$ and we present a result about the existence and uniqueness of such points. Moreover, as an application of our result, we study the problem of existence and uniqueness of solutions for a class of systems of functional equations arising in dynamic programming.

\section{Introduction}

The Banach contraction mapping principle is one of the pivotal results of analysis. It is widely considered as the source of metric fixed point theory. Also, its significance lies in its vast applicability in a great number of branches of mathematics and other sciences.

Generalizations of the above principle have been a heavily investigated branch of research. Particularly, one of these generalizations uses the so-called comparison functions. These functions are defined as functions $\varphi: \mathbb{R}_{+} \rightarrow \mathbb{R}_{+}$ which are increasing and satisfy $\varphi^{n}(t) \rightarrow 0$ when $n \rightarrow \infty$ for $t>0$, where $\varphi^{n}$ denotes the $n$-iteration of $\varphi$. Examples of such functions are $\varphi(t)=\lambda t$ with $\lambda \in(0,1), \varphi(t)=\arctan t$, $\varphi(t)=\ln (1+t)$, and $\varphi(t)=t /(1+t)$, among others.

The above-mentioned generalization of the Banach contraction mapping principle is the following result and it appears in $[1,2]$.

Theorem 1. Let $(X, d)$ be a complete metric space and let $T$ : $X \rightarrow X$ be a mapping satisfying $d(T x, T y) \leq \phi(d(x, y))$, for any $x, y \in X$, where $\phi$ is a comparison function. Then $T$ has a unique fixed point.

In this paper, we consider a nonempty set $S$ and by $B(S)$ we denote the set of all bounded real functions defined on $S$. According to the ordinary addition of functions and scalar multiplication and endowing with the norm $\|u\|=$ $\sup _{x \in S}|\mathcal{u}(x)|, B(S)$ is a Banach space.
Notice that the distance in $B(S)$ is defined as $d(u, v)=$ $\sup _{x \in S}\{|u(x)-v(x)|\}$, for $u, v \in B(S)$.

The aim of this paper is to present a result about the existence and uniqueness of an $\alpha$-coupled fixed point (see Section 2) in $B(S)$ and, as an application of this result, we will study the problem of existence and uniqueness of solutions of the following system of functional equations arising in dynamic programming:

$$
\begin{aligned}
& u(x)=\sup _{y \in D}\{g(x, y)+F(x, y, u(T(x, y)), v(T(x, y)))\} \\
& \begin{aligned}
v(x)=\sup _{y \in D}\{g(x, y) \\
\quad+F(x, y, u(\alpha(T(x, y))), v(\alpha(T(x, y))))\}
\end{aligned}
\end{aligned}
$$

under certain assumptions.

For further information about the functional equations appearing in dynamic programming, we refer the reader to [3-6].

\section{Generalized Coupled Fixed Point Theorem in $B(S)$}

Our starting point in this section is the definition of $\alpha$ coupled fixed point in $B(S)$. For this purpose, suppose that $S$ is a nonempty set and $\alpha: S \rightarrow S$ a mapping. 
Definition 2. An element $(u, v) \in B(S) \times B(S)$ is called an $\alpha$ coupled fixed point of a mapping $G: B(S) \times B(S) \rightarrow B(S)$ if $G(u, v)=u$ and $G(u \circ \alpha, v \circ \alpha)=v$.

The following theorem is the main result of the paper and it gives us a sufficient condition for the existence and uniqueness of an $\alpha$-coupled fixed point.

Theorem 3. Suppose that $\alpha: S \rightarrow S$ and $G: B(S) \times B(S) \rightarrow$ $B(S)$ are two mappings. If $G$ satisfies

$$
d(G(x, y), G(u, v)) \leq \phi(\max (d(x, u), d(y, v))),
$$

for any $x, y, u, v \in B(S)$, where $\phi$ is a comparison function, then $G$ has a unique $\alpha$-coupled fixed point.

Proof. Consider the Cartesian product $B(S) \times B(S)$ endowed with the distance defined by

$$
\bar{d}((x, y),(u, v))=\max (d(x, u), d(y, v)) .
$$

It is easily seen that $(B(S) \times B(S), \bar{d})$ is a complete metric space.

Now, we consider the mapping $\bar{G}: B(S) \times B(S) \rightarrow B(S) \times$ $B(S)$ defined by

$$
\bar{G}(x, y)=(G(x, y), G(x \circ \alpha, y \circ \alpha)) .
$$

Notice that if $x \in B(S)$ then $x \circ \alpha \in B(S)$.

Next, we check that $\bar{G}$ satisfies assumptions of Theorem 1 . In fact, according to (2), we have that for any $x, y, u, v \in B(S)$

$$
\begin{gathered}
\bar{d}(\bar{G}(x, y), \bar{G}(u, v)) \\
=\bar{d}((G(x, y), G(x \circ \alpha, y \circ \alpha)), \\
\quad(G(u, v), G(u \circ \alpha, v \circ \alpha))) \\
=\max \{d(G(x, y), G(u, v)), \\
d(G(x \circ \alpha, y \circ \alpha), G(u \circ \alpha, v \circ \alpha))\} \\
\leq \max \{\phi(\max (d(x, u), d(y, v))), \\
\phi(\max (d(x \circ \alpha, u \circ \alpha), d(y \circ \alpha, v \circ \alpha)))\} .
\end{gathered}
$$

Now, taking into account the definition of the distance on $B(S)$, we have

$$
\begin{aligned}
d(x \circ \alpha, u \circ \alpha) & =\sup _{s \in S}\{|(x \circ \alpha)(s)-(u \circ \alpha)(s)|\} \\
& =\sup _{s \in S}\{|x(\alpha(s))-u(\alpha(s))|\} \\
& \leq \sup _{s \in S}\{|x(s)-u(s)|\}=d(x, u)
\end{aligned}
$$

and, by a similar argument, we have $d(y \circ \alpha, v \circ \alpha) \leq d(y, v)$. Therefore, from (5) and (6), we get $\bar{d}(\bar{G}(x, y), \bar{G}(u, v)) \leq$ $\phi(\max (d(x, u), d(y, v)))=\phi(\bar{d}((x, y),(u, v)))$.

Therefore, Theorem 1 gives us the existence of a unique $\left(x_{0}, y_{0}\right) \in B(S) \times B(S)$ such that $\bar{G}\left(x_{0}, y_{0}\right)=\left(x_{0}, y_{0}\right)$ or, equivalently, $G\left(x_{0}, y_{0}\right)=x_{0}$ and $G\left(x_{0} \circ \alpha, y_{0} \circ \alpha\right)=y_{0}$.

This completes the proof.

\section{Application to Dynamic Programming}

The following types of systems of functional equations

$$
\begin{aligned}
u(x)=\sup _{y \in D}\{g(x, y)+F(x, y, u(T(x, y)), v(T(x, y)))\} \\
v(x)=\sup _{y \in D}\{g(x, y) \\
\quad+F(x, y, u(\alpha(T(x, y))), v(\alpha(T(x, y))))\}
\end{aligned}
$$

appear in the study of dynamic programming (see [7]), where $x \in S$ and $S$ is a state space, $D$ is a decision space, $T: S \times D \rightarrow$ $S, g: S \times D \rightarrow \mathbb{R}, F: S \times D \times \mathbb{R} \times \mathbb{R} \rightarrow \mathbb{R}$, and $\alpha: S \rightarrow S$ are given mappings.

The following theorem gives us a sufficient condition for the existence and uniqueness of solutions to problem (7).

Theorem 4. Suppose the following assumptions:

(i) $g: S \times D \rightarrow \mathbb{R}$ and $F(-,-, 0,0): S \times D \rightarrow \mathbb{R}$ are bounded functions;

(ii) there exists a comparison function $\varphi$ such that for any $x \in S, y \in D$ and $t, s, t_{1}, s_{1} \in \mathbb{R}, \mid F(x, y, t, s)-$ $F\left(x, y, t_{1}, s_{1}\right) \mid \leq \varphi\left(\max \left(\left|t-t_{1}\right|,\left|s-s_{1}\right|\right)\right)$.

Then, problem (7) has a unique solution $\left(u_{0}, v_{0}\right) \in B(S) \times B(S)$.

As a previous result for the proof of Theorem 4, we need the next lemma.

Lemma 5. Suppose that $H, G: S \rightarrow \mathbb{R}$ are two bounded functions. Then

$$
\left|\sup _{y \in S} H(y)-\sup _{y \in S} G(y)\right| \leq \sup _{y \in S}|H(y)-G(y)| .
$$

Proof. Obviously, this result is true when $\sup _{y \in S}\{H(y)\}=$ $\sup _{y \in S}\{G(y)\}$.

If we suppose that $\sup _{y \in S}\{H(y)\}>\sup _{y \in S}\{G(y)\}$ (same argument works if we suppose that $\sup _{y \in S}\{H(y)\}<$ $\left.\sup _{y \in S}\{G(y)\}\right)$ then for any $y_{0} \in S$

$$
H\left(y_{0}\right)-\sup _{y \in S}\{G(y)\} \leq H\left(y_{0}\right)-G\left(y_{0}\right) \leq\left|H\left(y_{0}\right)-G\left(y_{0}\right)\right|
$$

and, consequently,

$$
\sup _{y \in S}\left\{H(y)-\sup _{y \in S}\{G(y)\}\right\} \leq \sup _{y \in S}\{|H(y)-G(y)|\} \text {. }
$$

Since $\sup _{y \in S}\{H(y)-a\}=\sup _{y \in S}\{H(y)\}-a$, for any $a \in \mathbb{R}$, it follows

$$
\sup _{y \in S}\{H(y)\}-\sup _{y \in S}\{G(y)\} \leq \sup _{y \in S}\{|H(y)-G(y)|\}
$$

and this proves our claim. 
Proof of Theorem 4. Consider the operator $G$ defined on $B(S) \times B(S)$ as

$$
\begin{aligned}
G & (u, v)(x) \\
& =\sup _{y \in D}\{g(x, y)+F(x, y, u(T(x, y)), v(T(x, y)))\}
\end{aligned}
$$

for $(u, v) \in B(S) \times B(S)$ and $x \in S$. By assumptions (i) and (ii), we have

$$
\begin{aligned}
& |G(u, v)(x)| \\
& \leq \sup _{y \in D}|g(x, y)+F(x, y, u(T(x, y)), v(T(x, y)))| \\
& \leq \sup _{y \in D}|g(x, y)|+\sup _{y \in D}|F(x, y, u(T(x, y)), v(T(x, y)))| \\
& \leq \sup _{y \in D}|g(x, y)|+\sup _{y \in D}\{\mid F(x, y, u(T(x, y)), v(T(x, y))) \\
& \leq \sup _{y \in D}|g(x, y)| \\
& \quad+\sup _{y \in D}\{\varphi(\max (|u(T(x, y))|,|v(T(x, y))|)) \\
& \quad+|F(x, y, 0,0)|\} .
\end{aligned}
$$

According to assumption (i) and, since $(u, v) \in B(S) \times$ $B(S)$, we obtain that $G(u, v) \in B(S)$. Therefore, $G: B(S) \times$ $B(S) \rightarrow B(S)$.

Now, we check that $G$ satisfies condition (2) of Theorem 3. In fact, for any $u, v, u_{1}, v_{1} \in B(S)$, we have

$$
d\left(G(u, v), G\left(u_{1}, v_{1}\right)\right)=\sup _{x \in S}\left|G(u, v)(x)-G\left(u_{1}, v_{1}\right)(x)\right| .
$$

Then, from assumption (ii) and Lemma 5 and using the fact that $\varphi$ is an increasing function, for any $x \in S$, we have

$$
\begin{aligned}
& \left|G(u, v)(x)-G\left(u_{1}, v_{1}\right)(x)\right| \\
& =\mid \sup _{y \in D}\{g(x, y)+F(x, y, u(T(x, y)), v(T(x, y)))\} \\
& \quad-\sup _{y \in D}\left\{g(x, y)+F\left(x, y, u_{1}(T(x, y)), v_{1}(T(x, y))\right)\right\} \mid \\
& \leq \sup _{y \in D} \mid F(x, y, u(T(x, y)), v(T(x, y))) \\
& \quad-F\left(x, y, u_{1}(T(x, y)), v_{1}(T(x, y))\right) \mid \\
& \leq \varphi\left(\operatorname { m a x } \left\{\left|u(T(x, y))-u_{1}(T(x, y))\right|,\right.\right. \\
& \left.\left.\quad\left|v(T(x, y))-v_{1}(T(x, y))\right|\right\}\right) \\
& \leq \varphi\left(\max \left(\left\|u-u_{1}\right\|,\left\|v-v_{1}\right\|\right)\right) \\
& =\varphi\left(\max \left(d\left(u, u_{1}\right), d\left(v, v_{1}\right)\right)\right) .
\end{aligned}
$$

Therefore, condition (2) of Theorem 3 is satisfied and, consequently, $G$ has a unique $\alpha$-coupled fixed point $\left(u_{0}, v_{0}\right) \in$ $B(S) \times B(S)$. This means that $G\left(u_{0}, v_{0}\right)=u_{0}$ and $G\left(u_{0} \circ \alpha, v_{0} \circ\right.$ $\alpha)=v_{0}$ or, equivalently, for $x \in S$,

$$
\begin{aligned}
u_{0}(x)=\sup _{y \in D}\{g(x, y) \\
\left.\quad+F\left(x, y, u_{0}(T(x, y)), v_{0}(T(x, y))\right)\right\}, \\
v_{0}(x)=\sup _{y \in D}\{g(x, y) \\
\left.+F\left(x, y, u_{0}(\alpha(T(x, y))), v_{0}(\alpha(T(x, y)))\right)\right\} .
\end{aligned}
$$

This completes the proof.

In order to illustrate our results, we present the following example.

Consider the following system of functional equations, where $x \in[0,1]$ :

$$
\begin{aligned}
& u(x)=\sup _{y \in \mathbb{R}}\left\{e^{-(x+|y|)}\right. \\
& +\arctan \left(\frac{1}{2}(x+|y|+|u(|\sin (x+y)|)|\right. \\
& +|v(|\sin (x+y)|)|))\}, \\
& v(x)=\sup _{y \in \mathbb{R}}\left\{e^{-(x+|y|)}\right. \\
& \begin{array}{r}
+\arctan \left(\frac { 1 } { 2 } \left(x+|y|+\left|u\left(\frac{1}{1+|\sin (x+y)|}\right)\right|\right.\right. \\
\left.\left.\left.+\left|v\left(\frac{1}{1+|\sin (x+y)|}\right)\right|\right)\right)\right\} .
\end{array}
\end{aligned}
$$

This system appears in dynamic programming, where the state space is $S=[0,1]$ and the decision space is $D=\mathbb{R}$.

Notice that the system (17) is a particular case of (7), where $S=[0,1], D=\mathbb{R}, g:[0,1] \times \mathbb{R} \rightarrow \mathbb{R}$ is defined as $g(x, y)=e^{-(x+|y|)}, \alpha:[0,1] \rightarrow[0,1]$ is given by $\alpha(t)=$ $1 /(1+t), T:[0,1] \times \mathbb{R} \rightarrow[0,1]$ is $T(x, y)=|\sin (x+y)|$, and $F:[0,1] \times \mathbb{R} \times \mathbb{R} \times \mathbb{R} \rightarrow \mathbb{R}$ is defined as

$$
F(x, y, t, s)=\arctan \left(\frac{1}{2}(x+|y|+|t|+|s|)\right) .
$$

Notice that $|g(x, y)| \leq 1$ and $|F(x, y, 0,0)|=$ $|\arctan ((1 / 2)(x+|y|))| \leq \pi / 2$.

Therefore, assumption (i) of Theorem 4 is satisfied. 
On the other hand, for $x \in[0,1]$ and $y, t, s, t_{1}, s_{1} \in \mathbb{R}$, we have

$$
\begin{aligned}
& \left|F(x, y, t, s)-F\left(x, y, t_{1}, s_{1}\right)\right| \\
& =\mid \arctan \left(\frac{1}{2}(x+|y|+|t|+|s|)\right) \\
& \quad \quad-\arctan \left(\frac{1}{2}\left(x+|y|+\left|t_{1}\right|+\left|s_{1}\right|\right)\right) \mid \\
& \quad \leq \arctan \left(\left|\frac{1}{2}\left(|t|-\left|t_{1}\right|+|s|-\left|s_{1}\right|\right)\right|\right) \\
& \leq \arctan \left(\frac{1}{2}\left(|| t|-| t_{1}||+|| s|-| s_{1}||\right)\right) \\
& \leq \arctan \left(\frac{1}{2}\left(\left|t-t_{1}\right|+\left|s-s_{1}\right|\right)\right) \\
& \leq \arctan \left(\frac{1}{2}\left(2 \max \left\{\left|t-t_{1}\right|,\left|s-s_{1}\right|\right\}\right)\right) \\
& =\arctan \left(\max \left\{\left|t-t_{1}\right|,\left|s-s_{1}\right|\right\}\right),
\end{aligned}
$$

where we have used the nondecreasing character of the function $\varphi(t)=\arctan (t)$ and the fact that $\mid \arctan t-$ $\arctan s \mid \leq \arctan (|t-s|)$, for any $t, s \in \mathbb{R}_{+}$.

It is easily seen that $\varphi(t)=\arctan (t)$, for $t \geq 0$, is a comparison function and, therefore, assumption (ii) of Theorem 4 is satisfied.

By Theorem 4, the system (17) has a unique solution $\left(u_{0}, v_{0}\right) \in B([0,1]) \times B([0,1])$.

\section{Conflict of Interests}

The authors declare that there is no conflict of interests regarding the publication of this paper.

\section{References}

[1] J. Matkowski, "Integrable solutions of functional equations," Dissertationes Mathematicae, vol. 127, pp. 1-68, 1975.

[2] V. Berinde, Iterative Approximation of Fixed Points, vol. 1912 of Lecture Notes in Mathematics, Springer, Berlin, Germany, 2nd edition, 2007.

[3] Z. Liu, R. P. Agarwal, and S. M. Kang, "On solvability of functional equations and system of functional equations arising in dynamic programming," Journal of Mathematical Analysis and Applications, vol. 297, no. 1, pp. 111-130, 2004.

[4] Z. Liu, J. S. Ume, and S. M. Kang, "Some existence theorems for functional equations and system of functional equations arising in dynamic programming," Taiwanese Journal of Mathematics, vol. 14, no. 4, pp. 1517-1536, 2010.

[5] Z. Liu, J. S. Ume, and S. M. Kang, "On properties of solutions for two functional equations arising in dynamic programming," Fixed Point Theory and Applications, vol. 2010, Article ID 905858, 19 pages, 2010.

[6] Deepmala, "Existence theorems for solvability of a functional equation arising in dynamic programming," International Journal of Mathematics and Mathematical Sciences, vol. 2014, Article ID 706585, 9 pages, 2014.
[7] R. Bellman and E. S. Lee, "Functional equations in dynamic programming," Aequationes Mathematicae, vol. 17, no. 1, pp. 1$18,1978$. 


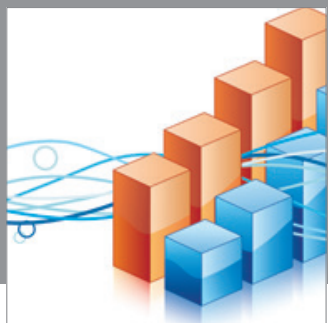

Advances in

Operations Research

mansans

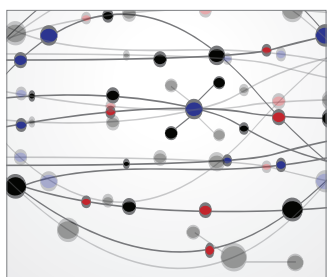

The Scientific World Journal
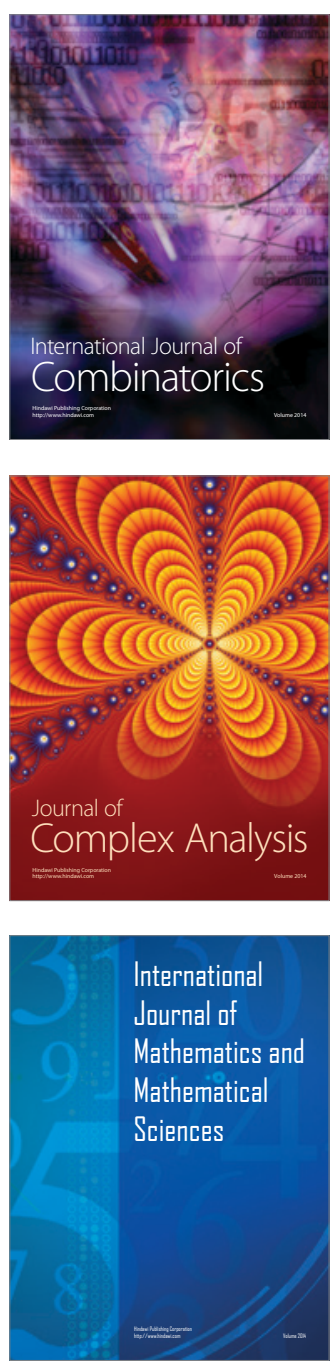
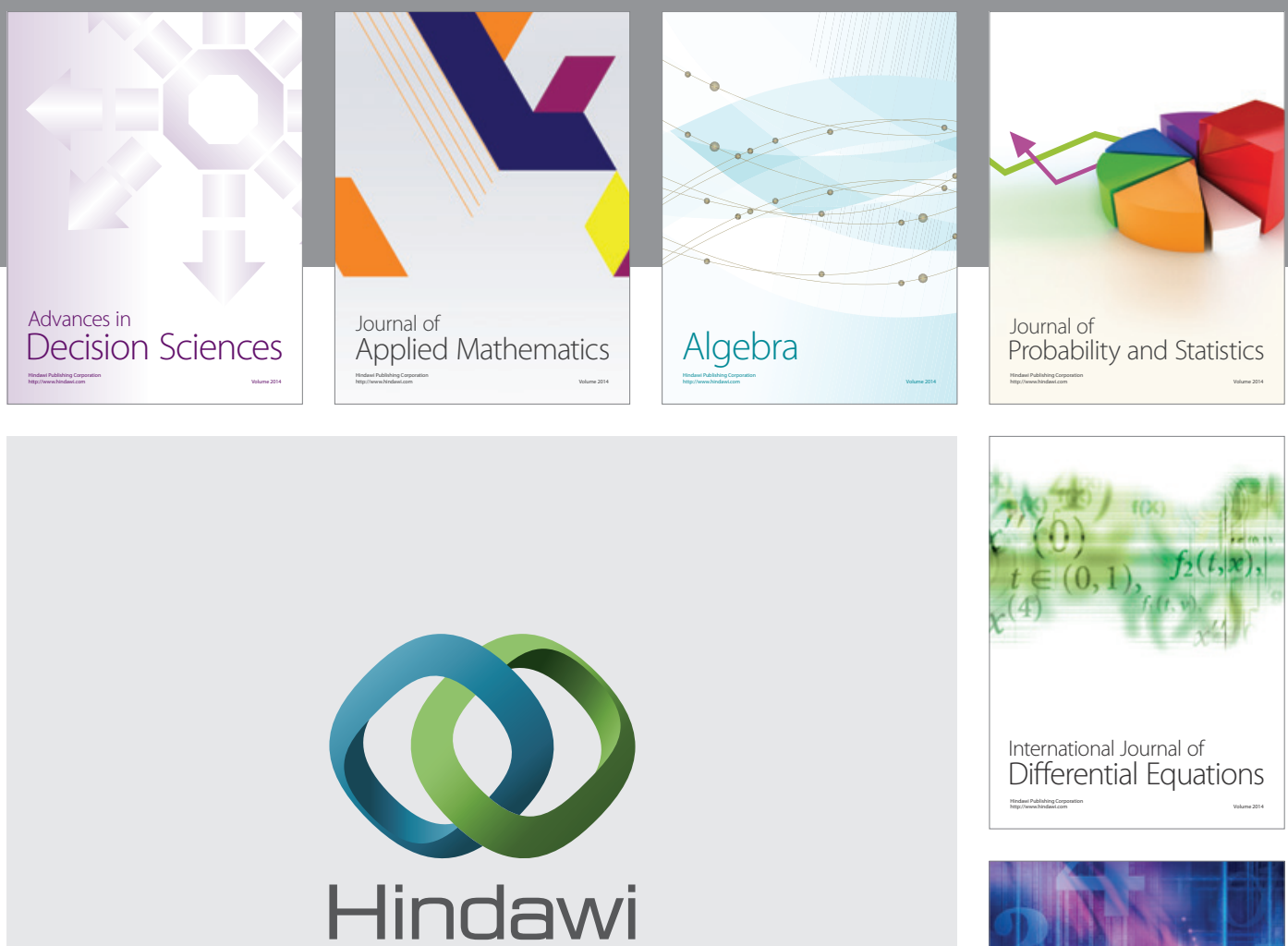

Submit your manuscripts at http://www.hindawi.com
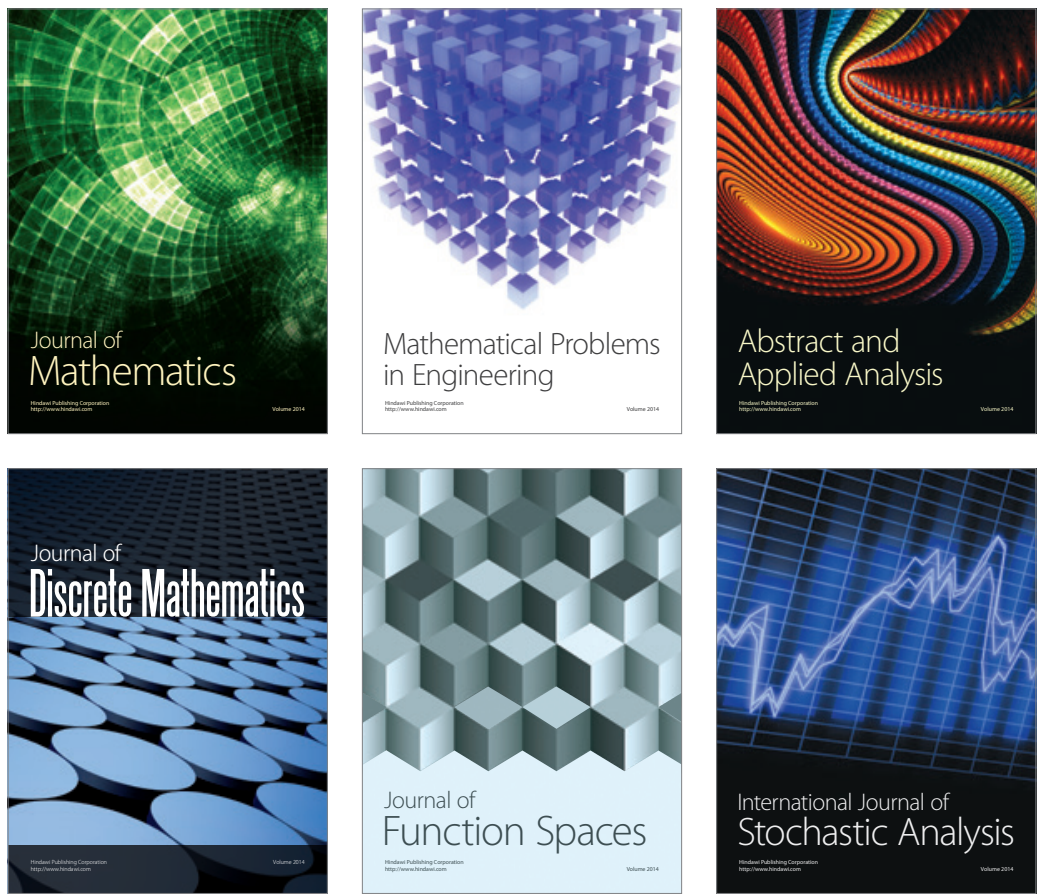

Journal of

Function Spaces

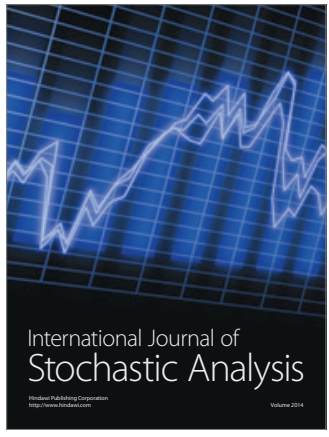

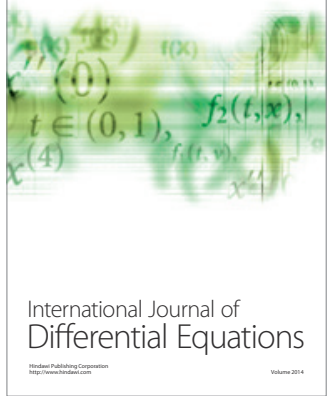
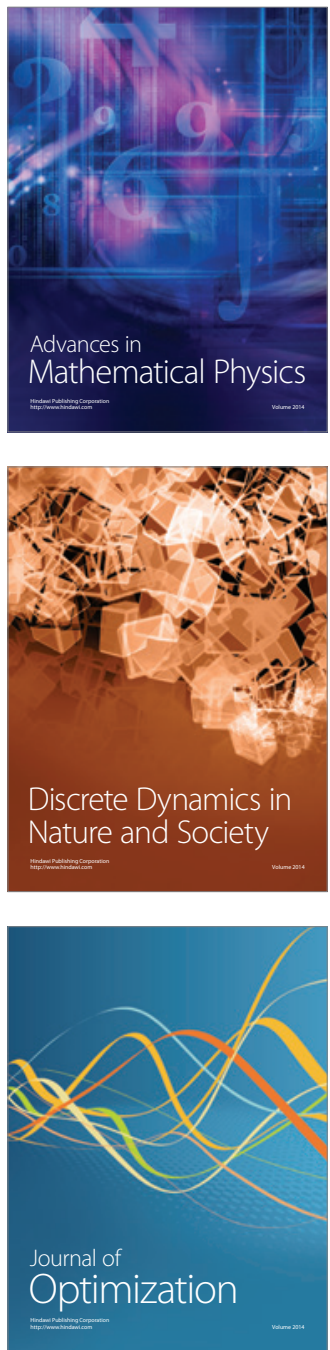